\title{
Raman Spectroscopic Nano-imaging of Optical Fields of Metal Nanostructures with a Chemically Modified Metallic Tip
}

\author{
Ryo Kato ${ }^{1,2}$, Takayuki Umakoshi ${ }^{1,3,4}$ and Prabhat Verma ${ }^{1 *}$ \\ ${ }^{1}$ Department of Applied Physics, Osaka University, Suita, Osaka 565-0871, Japan \\ ${ }^{2}$ Institute of Post-LED Photonics, Tokushima University, 2-1 Minamijosanjima-cho, Tokushima, \\ Tokushima 770-8506, Japan \\ ${ }^{3}$ Institute for Advanced Co-Creation Studies, Osaka University \\ ${ }^{4}$ PRESTO, Japan Science and Technology Agency, Kawaguchi, Saitama 332- 0012, Japan \\ *Corresponding Author, Email: verma@ap.eng.osaka-u.ac.jp
}

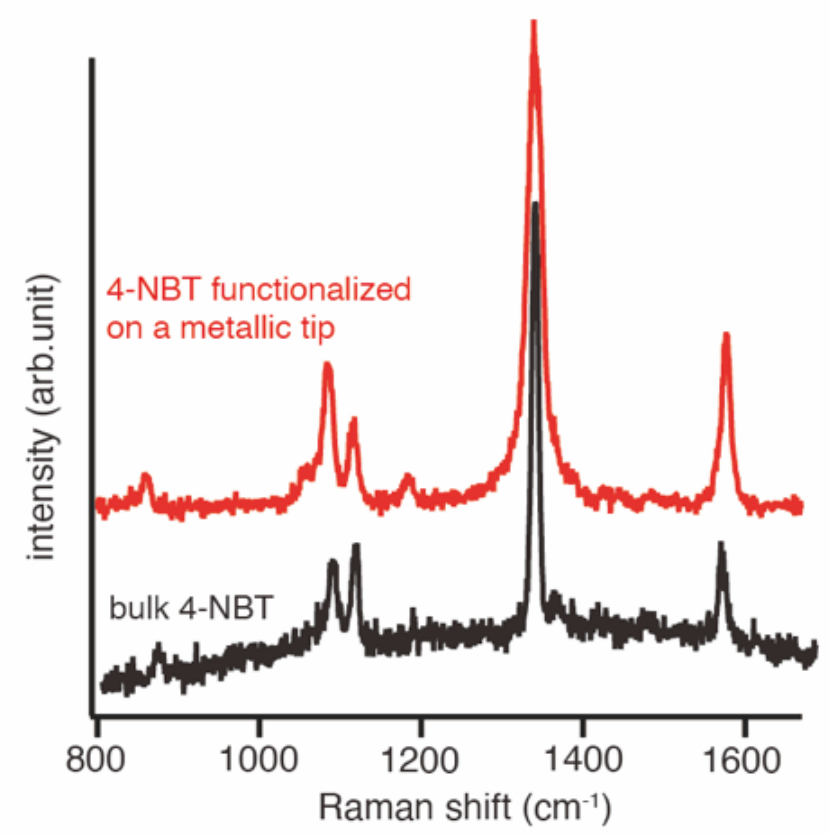

Figure S1. Raman spectra of 4-NBT molecules functionalized on a metallic tip (red) and bulk 4-NBT (black).

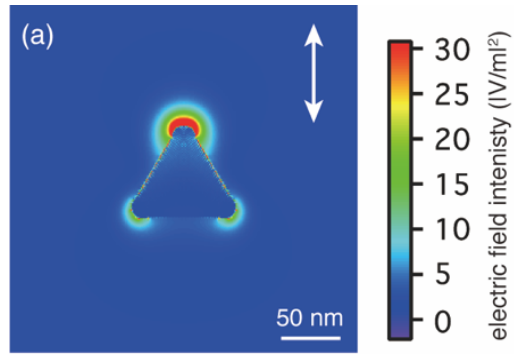

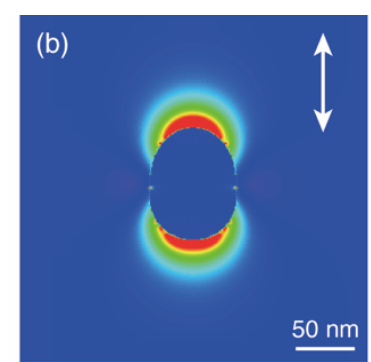

Figure S2. FDTD simulation of plasmonically enhanced electric field intensity distribution of (a) a gold nanotriangle and (b) a gold elliptical structure. White arrows indicate the direction of incident polarization. 
(a)

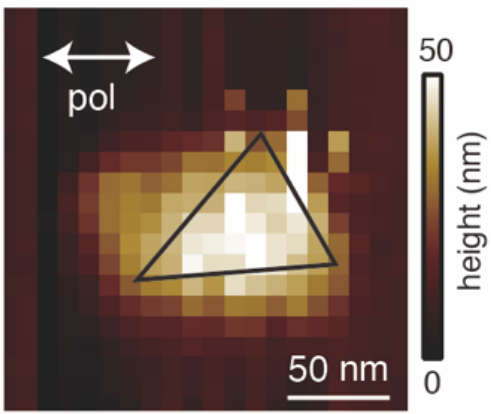

b)

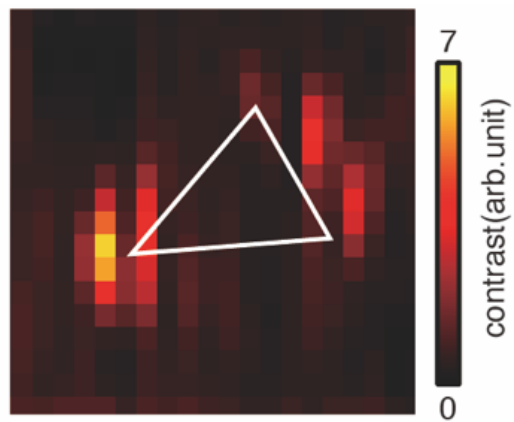

Figure S3. Simultaneously recorded AFM image and TERS contrast image of a plasmonic Au nanostructure, constructed by Raman mode at $1350 \mathrm{~cm}^{-1}$ with an incident polarization perpendicular to the one shown in Figure 2 in main text.

(a)

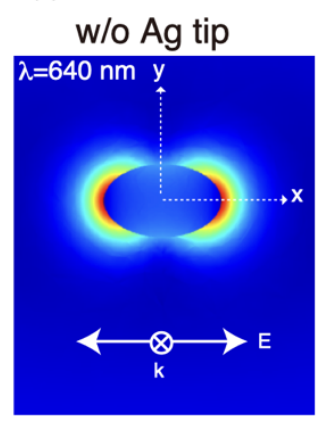

w/ Ag tip

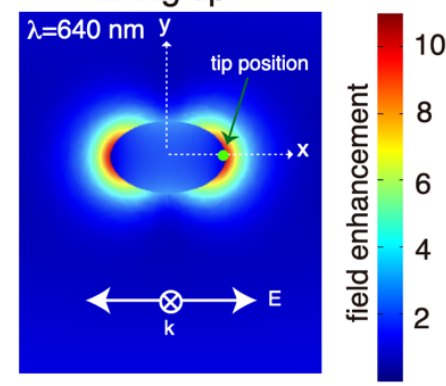

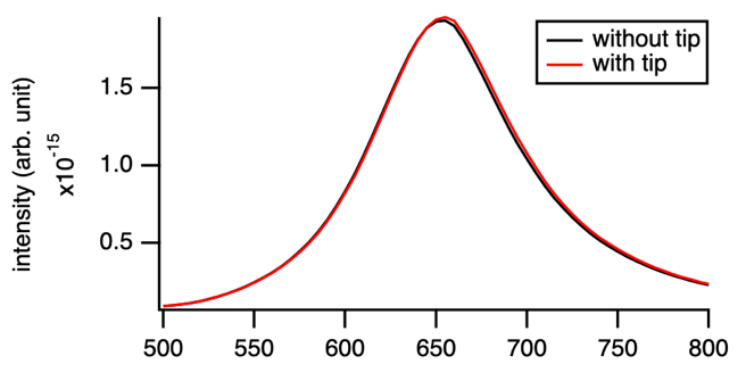

Figure S4. Simulation results showing that the presence of a tip (Au nanorod) in contact with metal nanostructure does not significantly affect plasmonic properties of the nanostructure. (a) Comparison of simulated field distribution of a metallic nanostructure without and with a metallic tip in contact. (b) Comparison of simulated LSPR spectra of metallic nanostructure without and with a metallic tip.

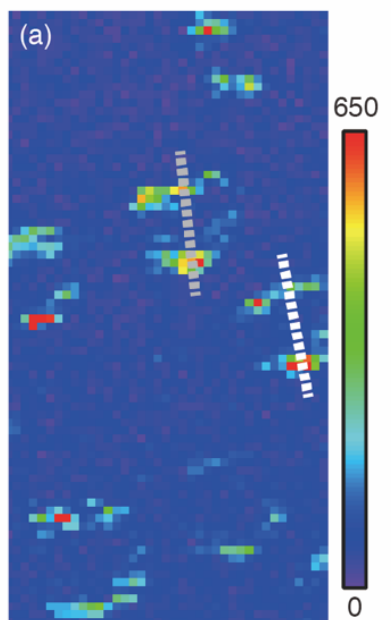

(b)

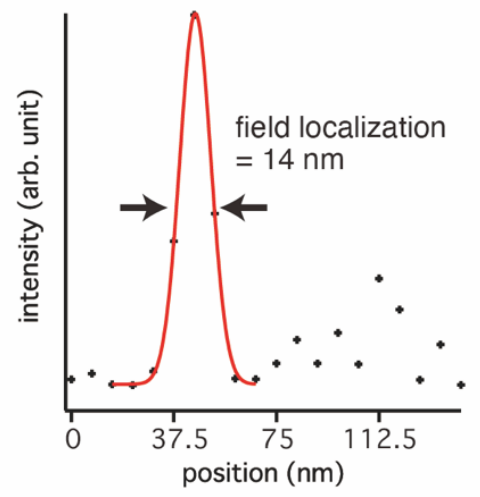

(c)

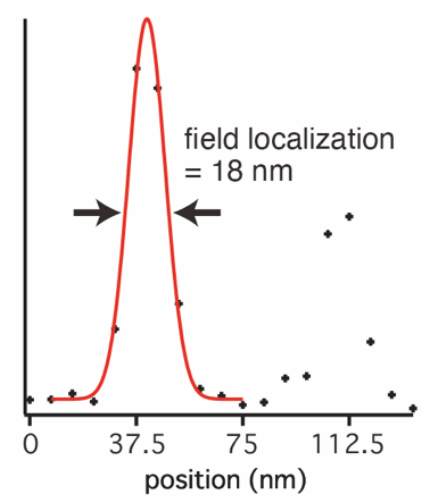

Figure S5. (a) TERS intensity image of gold nanostructures obtained from Raman signal at $1580 \mathrm{~cm}^{-1}$. Line-profiles of TERS intensity along the gray (b) and the white (c) dotted lines shown in (a). 


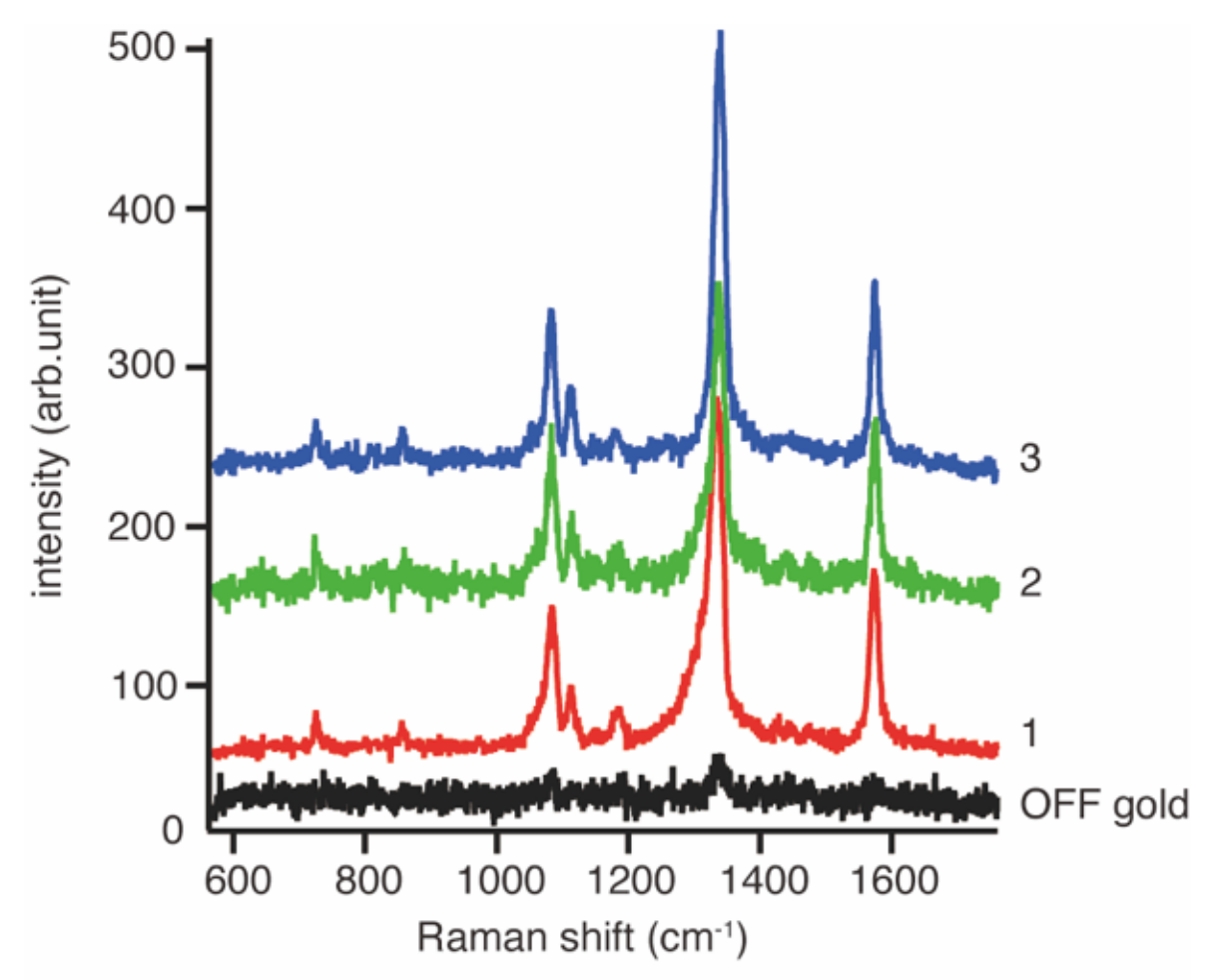

Figure S6. TERS spectra of a 4-NBT functionalized tip recorded on Au nanostructures at positions 1, 2 and 3, respectively, marked in Figure 2(b) in the main text. The black spectrum shows TERS spectrum measured at a position far from the Au nanostructures. The absence of a peak at $1450 \mathrm{~cm}^{-1}$ confirms that 4-NBT molecules do not transform into 4-ABT molecules under the present experimental conditions. 